\title{
Mycoplasma sualvi, a New Species from the Intestinal and Urogenital Tracts of Pigs
}

\author{
R. N. GOURLAY, ${ }^{1}$ SARA G. WYLD, ${ }^{1}$ AND R. H. LEACH ${ }^{2}$ \\ ARC Institute for Research on Animal Diseases, Compton, Newbury, Berkshire, England, ${ }^{1}$ and Mycoplasma
} Reference Laboratory, Public Health Laboratory Service, Norwich, NR2 3TX, England ${ }^{2}$

Eight similar Mycoplasma isolates were obtained from rectal, intestinal, and vaginal specimens from pigs. Two indistinguishable clones of one isolate (Mayfield) were examined in detail. Although closely resembling the cattle organism Mycoplasma alvi in cultural and biochemical properties, the Mayfield clones differed from this species in morphological appearance and deoxyribonucleic acid base composition. The clones were serologically distinct from $M$. alvi and 58 currently recognized Mycoplasma species and subspecies, including all of those having similar biochemical properties or the same host. The Mayfield strain clone B (NCTC 10170) is designated the type strain of a new species, $M$. sualvi, which includes five of the seven other Mycoplasma isolates from pigs. The remaining two isolates appear to be serologically dissimilar from the Mayfield strain.

Three strains of Mycoplasma were isolated from porcine rectal swabs, and the partial characterization of one of them (the Mayfield strain) suggested that they might represent a new species (1). This paper presents a detailed characterization of two clones of the Mayfield strain and proposes their classification, together with five other similar isolates from pigs, as a new Mycoplasma species.

\section{MATERIALS AND METHODS}

Mycoplasma strains studied. The Mayfield strain was isolated from a porcine rectal swab as previously described (1). Before testing, it was "filtercloned" three times (2), and two separate clones (A and B) were examined for characterization purposes.

Strain H770 was isolated from the vagina, strain 2495 was isolated from the colon, strains PF1 and PF3 were isolated from the rectum, and strains 1828,2284 , and 2685 were isolated from the small intestine of pigs.

The other Mycoplasma species and antisera used in comparative studies are listed in Table 1.

Media. Except where indicated, U3/7.0 broth and U3 agar plates $(2,3)$ were used. The main ingredients of these media are fetal calf serum, Hartley digest broth, and yeast extract.

Methods. Except where otherwise indicated in the text, all materials and methods were as described for the isolation and characterization of Mycoplasma alvi (2).

\section{RESULTS}

Cultural characteristics and reversion studies. The Mayfield clones had cultural characteristics similar to those of the bovine isolate M. alvi (2). When subcultured in U3/7.0 broth, the Mayfield clones produced both opacity and an acid $\mathrm{pH}$ color change ( $1.5 \mathrm{pH}$ units) after 3 to
4 days of incubation at $37^{\circ} \mathrm{C}$. Unlike $M$. alvi, the porcine strains produced an acid color change $(0.8 \mathrm{pH}$ unit) in glucose serum (GS) broth $(\mathrm{pH}$ 7.8) (3). However, acidification in the U3/7.0 broth occurred more rapidly than in GS broth. In arginine broth (2), the porcine strains produced an initial acid color change (1.5 $\mathrm{pH}$ units) after 3 to 6 days of incubation, followed after 2 to 3 weeks by an alkaline reaction manifested by a return to the original $\mathrm{pH}$ of the medium $(\mathrm{pH}$ 7.0). The Mayfield clones grew less rapidly in $\mathrm{U} 3 / 7.0$ broth at $30^{\circ} \mathrm{C}$ than at $37^{\circ} \mathrm{C}$, and no color change was observed after 70 days of incubation at $25^{\circ} \mathrm{C}$.

Both Mayfield clones produced typical $M y$. coplasma colonies on U3 agar after about 4 days of aerobic incubation at $37^{\circ} \mathrm{C}$. On GS agar, colonies usually developed below the surface of the agar. Studies on the growth of the porcine strains on $\mathrm{U} 3 / 7.0$ agar at $37^{\circ} \mathrm{C}$ under different gaseous conditions revealed that they grew both aerobically and anaerobically, but the largest colonies were on plates incubated anaerobically with $10 \%$ added $\mathrm{CO}_{2}$.

Reversion studies with these strains, involving subculture to $10^{-7}$ in U3/7.0 broth free from ampicillin and thallous acetate followed by a further five passages on inhibitor-free agar medium, revealed no colonial, cultural, or morphological evidence of reversion to cell-walled bacterial forms.

Sterol requirement and biochemical characteristics. Like $M$. alvi (2), the Mayfield clones were digitonin susceptible and polyanetholsulfonate resistant, and they required sterol for growth, did not form "film and spots" or reduce tetrazolium aerobically, and did not uti- 
TABl.E 1. Mycoplasma, Acholeplasma, and Anaeroplasma strains and antisera used in serological tests

\begin{tabular}{|c|c|}
\hline Mycoplasma, Acholeplasma, and Anaeroplasma species (and strains) & Antisera sources ${ }^{\prime \prime}$ \\
\hline 1. Mycoplasma sp. (Mayfield) & IRAD \\
\hline 2. Mycoplasma hyopneumoniae (J) & MRL \\
\hline 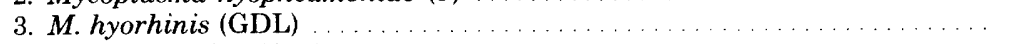 & MRL \\
\hline 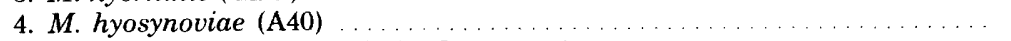 & M. Gois \\
\hline 5. Acholeplasma laidlawii (PG8, M1305/68) . . . . . . . . . . . . & MRL and IRAD \\
\hline 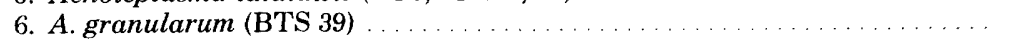 & MRL \\
\hline 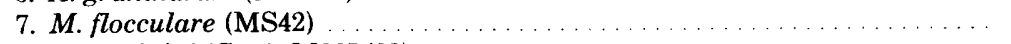 & N. F. Friis \\
\hline 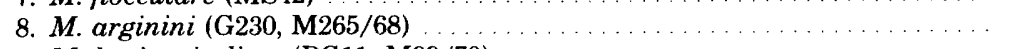 & MRL \\
\hline 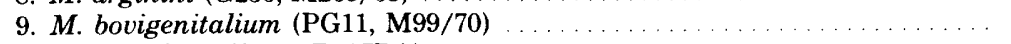 & MRL and IRAD \\
\hline 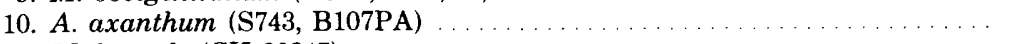 & IMM \\
\hline 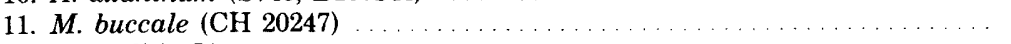 & MRL \\
\hline 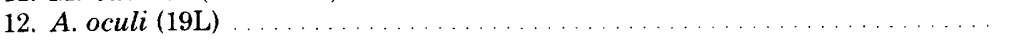 & R. M. Lemcke \\
\hline 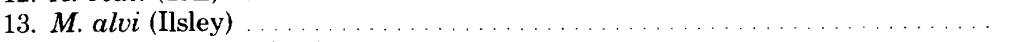 & IRAD \\
\hline$\ldots \ldots \ldots \ldots \ldots \ldots \ldots \ldots$ & IRAD \\
\hline 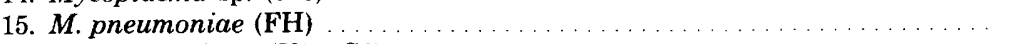 & MRL \\
\hline 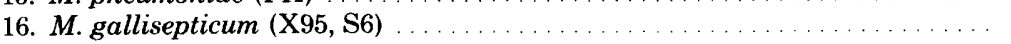 & MRL and IRAD \\
\hline 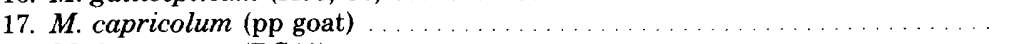 & R. M. Lemcke \\
\hline 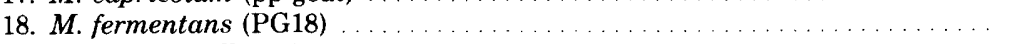 & MRL \\
\hline 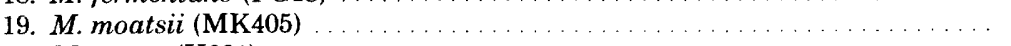 & IMM \\
\hline$\ldots \ldots \ldots \ldots \ldots \ldots \ldots \ldots \ldots \ldots \ldots \ldots$ & \\
\hline 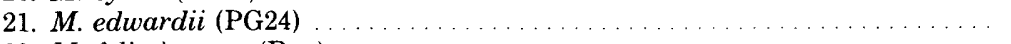 & \\
\hline$\ldots \ldots \ldots \ldots \ldots \ldots \ldots \ldots \ldots \ldots \ldots \ldots$ & \\
\hline 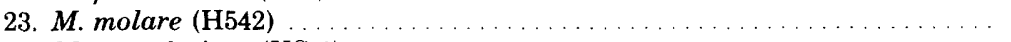 & \\
\hline 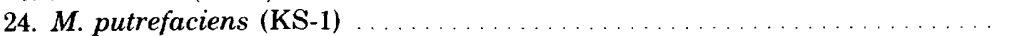 & \\
\hline 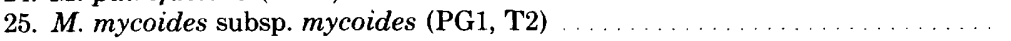 & MRL and IRAD \\
\hline 26. $M$. dispar $(462 / 2)$ & IRAD \\
\hline 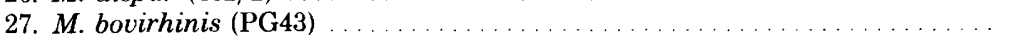 & MRL and IRAD \\
\hline 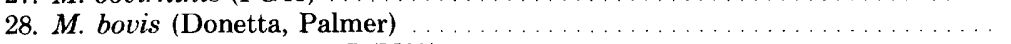 & MRL and IRAD \\
\hline 29. Mycoplasma sp. group 7 (N29) & MRL \\
\hline 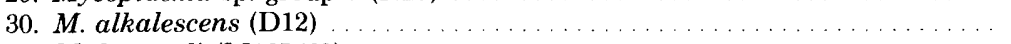 & MRL \\
\hline 31. M. bovoculi $(\mathrm{M} 165 / 69)$ & MRL and IRAD \\
\hline 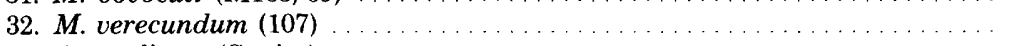 & IRAD \\
\hline 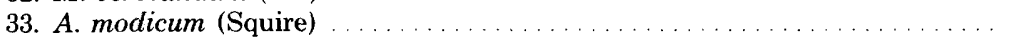 & MRL \\
\hline 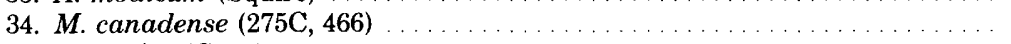 & H. L. Ruhnke \\
\hline 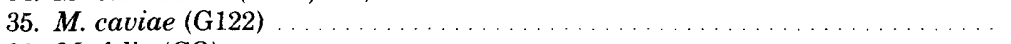 & IMM \\
\hline$\ldots \ldots \ldots \ldots \ldots \ldots \ldots \ldots \ldots \ldots$ & MRL \\
\hline 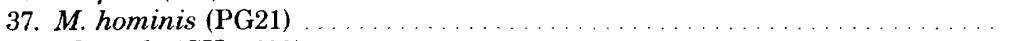 & MRL \\
\hline 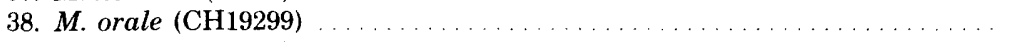 & MRL \\
\hline 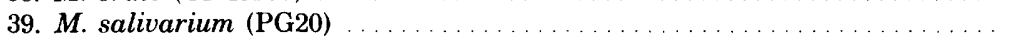 & MRL \\
\hline$\ldots \ldots \ldots \ldots \ldots$ & MRL \\
\hline$\ldots \ldots \ldots \ldots \ldots \ldots \ldots$ & MRL \\
\hline 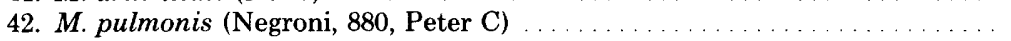 & MRL and IRAD \\
\hline 43. $M$. neurolyticum (Type A) & R. M. Lemcke \\
\hline 44. $M$. gallinarum $(\mathrm{PG} 16) \ldots$ & MRL \\
\hline 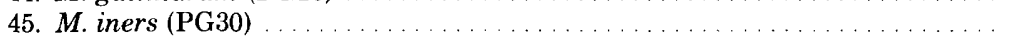 & NIH \\
\hline 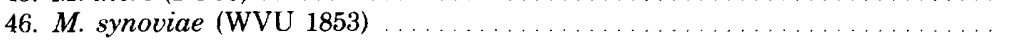 & N. Olson \\
\hline 47. $M$. meleagridis (17529) & NIH \\
\hline 48. $M$. anatis (1340) & NIH \\
\hline 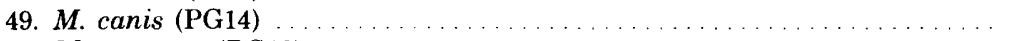 & MRL \\
\hline 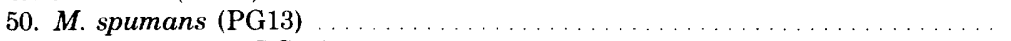 & M. Gois \\
\hline 51. M. maculosum (PG15) & M. Gois \\
\hline 52. M. equirhinis $(432 / 72)$ & MRL \\
\hline 53. $M$. agalactiae (PG2) & MRL \\
\hline 54. $M$. ovipneumoniae (M133/68) & MRL \\
\hline 55. M. mycoides subsp. capri (PG3) & MRL \\
\hline$\ldots \ldots \ldots \ldots \ldots \ldots$ & MRL \\
\hline 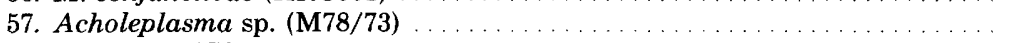 & MRL \\
\hline 58. M. gateae (CS) & M. Gois \\
\hline 59. Anaeroplasma bactoclasticum (JR, 7LA) $\ldots \ldots \ldots \ldots$ & I. Robinson \\
\hline 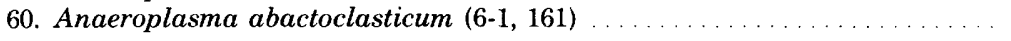 & I. Robinson \\
\hline
\end{tabular}

${ }^{a}$ Abbreviations: IRAD, Institute for Research on Animal Diseases, Compton, Berkshire, England; MRL, Mycoplasma Reference Laboratory, Public Health Laboratory Service, Norwich, England; NIH, National Institutes of Health, Bethesda, Md. (NIH Research Reference Reagents); IMM, Institute of Medical Microbiology, FAO/WHO Collaborating Center for Animal Mycoplasmas, University of Aarhus, Aarhus, Denmark. 
lize urea but metabolized both glucose and arginine.

DNA base composition. The guanine-pluscytosine $(\mathrm{G}+\mathrm{C})$ content of the deoxyribonucleic acid (DNA) of the Mayfield strain (clone B) obtained on two separate occasions was 23.7 mol\%.

Filtration studies. A culture of strain Mayfield clone $\mathrm{B}$ contained $7.4 \log _{10}$ colony-forming units (CFU) per $\mathrm{ml}$ before filtration; after filtration of 1-ml samples through membrane filters (Millipore Corp.) of 650-nm, 450-nm, 220-nm, and $100-\mathrm{nm}$ pore diameter, the counts were 6.4 , $6.35,<2.4$, and $<1.4 \log _{10}$ CFU, respectively. Similar results were obtained with the other Mayfield clone.

Morphology. In stained smears examined with the light microscope, cells of the Mayfield clones were mainly coccobacillary and resembled those of $M$. alvi (2). With the electron microscope, negatively stained culture deposits revealed flask- or club-shaped bodies, many of them elongated, resembling those of $M$. alvi (2). In thin sections, terminal structures were observed, but they were less well defined than those of $M$. alvi (Fig. 1). There was no densely staining central core, as seen with $M$. alvi, in preparations stained either with uranyl acetate or ruthenium red. A well-defined, triple-layered unit membrane was visible, but there was no evidence of a cell wall.

Serology. The Mayfield strain clones A and $B$ gave negative results with the growth inhibition (GI) and immunofluorescence (IFA) tests, and clone $A$ also gave negative results with the metabolism inhibition (MI) test, when tested against rabbit antisera to each of the 11 recognized species of Mycoplasma and Acholeplasma that have been isolated from pigs, namely, $M$. hyopneumoniae, $M$. hyorhinis, $M$. hyosynoviae, A. laidlawii, $A$. granularum, $M$. flocculare, $M$. arginini, $M$. bovigenitalium, $A$. axanthum, $M$. buccale, and $A$. oculi. Also yielding negative results were the morphologically similar $M$. alvi, $M$. pneumoniae, and $M$. gallisepticum and the biochemically similar $\boldsymbol{M}$. capricolum, $\boldsymbol{M}$. fermentans, and $M$. moatsii. Negative results were also obtained by the MI test when antisera to the remaining 36 mycoplasmas to which antisera were available (numbers 25 to 60 , Table 1) were tested against Mayfield strain clone A.

Antiserum prepared in a goat against Mayfield

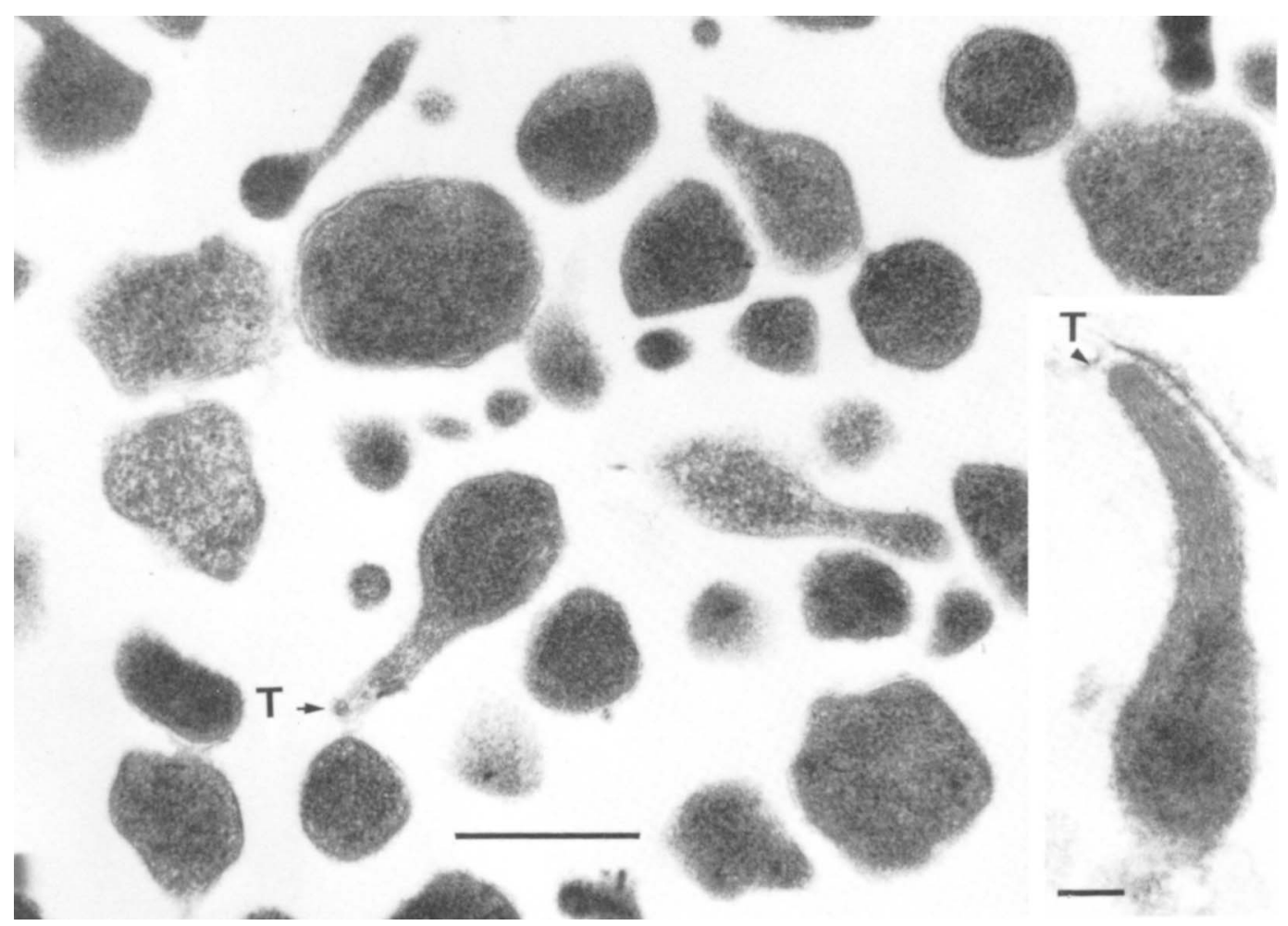

Fig. 1. Electron micrograph of cells of strain Mayfield; 3-day-old culture, thin-section preparation, stained with uranyl acetate. Some flask-or club-shaped bodies are visible with terminal structures (T). Bar represents $500 \mathrm{~nm}$. Insert: Higher magnification showing same structures. Bar represents $100 \mathrm{~nm}$. 
clone $\mathrm{B}$ gave positive results in the GI, MI, and IFA tests against the homologous organism, Mayfield clone A, and five of the seven other porcine isolates ( $\mathrm{PF} 1, \mathrm{H} 770,2284,2495$, and 2685). The antiserum gave negative results against the other two isolates (PF3 and 1828). This antiserum gave negative results by the GI, IFA, and MI tests against the above-mentioned morphologically or biochemically similar species $M$. pneumoniae, $M$. gallisepticum, $M$. alvi, $M$. capricolum, $M$. fermentans, and $M$. moatsii. The antiserum to the Mayfield strain also gave negative results by the MI, GI, and IFA tests against $\boldsymbol{M}$. cynos, $\boldsymbol{M}$. edwardii, $\boldsymbol{M}$. feliminutum (GI and IFA only), M. molare, and M. putrefaciens, species for which antisera were not available.

\section{DISCUSSION}

The Mayfield clones closely resemble $M$. alvi (2) in their cultural, morphological, and biochemical characteristics. There are, however, certain differences, some minor but others of major taxonomic importance. The important differences are as follows: (i) the DNA base composition of the Mayfield clones $(23.7 \mathrm{~mol} \%$ $\mathrm{G}+\mathrm{C})$ differs significantly from the value for $M$. alvi $(26.4 \mathrm{~mol} \%)$, (ii) there are morphological differences, particularly in their terminal structures as seen by electron microscopy, and (iii) conclusively, the Mayfield clones and $M$. alvi were serologically quite distinct from each other with respect to the tests employed.

As discussed previously in greater detail with regard to $M$. alvi (2), the Mayfield strain's colonial and cultural characteristics, lack of cell wall, failure to revert to cell wall forms, ability to pass through a $450-\mathrm{nm}$ membrane filter, low DNA base ratio $(23.7 \mathrm{~mol} \% \mathrm{G}+\mathrm{C})$, and sterol dependence conform to the requirements for inclusion within the order Mycoplasmatales and family Mycoplasmataceae (4). Its general biological properties (ability to grow aerobically, in contrast to Anaeroplasma spp., coccobacilliform morphology, in contrast to Spiroplasma spp., and failure to utilize urea, in contrast to Ureaplasma spp.) place it in the genus Mycoplasma.

The Mayfield strain clones were serologically distinct from 58 recognized species and subspe- cies of the order Mycoplasmatales, including all known porcine Mycoplasma species and all species with similar biochemical and cultural characteristics, including $M$. alvi. Since these mycoplasmas include virtually all the relevant named species required to be tested for taxonomic purposes (4), it is concluded that the Mayfield clones and the five other serologically similar isolates (PF1, H770, 2284, 2495, and 2685) represent a new species, for which the name $M y$. coplasma sualvi is proposed (su.al'vi. L.n. sus the hog; L.n. alvus bowel, womb, stomach; M.L. gen. $n$. sualvi of the bowel of the hog). The Mayfield strain (clone B) is designated the type strain of this species, and a culture of this strain has been deposited in the National Collection of Type Cultures under the number NCTC 10170.

Although isolates PF3 and 1828 possessed cultural, biological, and morphological characteristics similar to those of the Mayfield strain, they gave negative results in the GI, MI, and IFA tests against antiserum prepared to the Mayfield strain. Further work is required to determine the relationship of PF3 and 1828 to each other, to the Mayfield strain, and to other mycoplasmas.

\section{ACKNOWLEDGMENTS}

We thank I. Robinson and N. F. Friis for antisera to and for strains of Anaeroplasma sp. and $M$. flocculare, respectively; A. P. Bland for electron microscopy; and D. H. Pocock for carrying out the base composition estimations.

\section{REPRINT REQUESTS}

Address reprint requests to: Dr. R. N. Gourlay, Institute for Research on Animal Diseases, Compton, Nr. Newbury, Berkshire, England.

\section{LTTERATURE CITED}

1. Gourlay, R. N., and S. G. Wyld. 1976. Isolation of mycoplasmas from porcine rectal swabs. Br. Vet. J. 132:652-653.

2. Gourlay, R. N., S. G. Wyld, and R. H. Leach. 1977. Mycoplasma alvi, a new species from the bovine intestinal and urogenital tracts. Int. J. Syst. Bacteriol. 27:86-96.

3. Howard, C. J., J. Brownlie, R. N. Gourlay, and J. Collins. 1975. Presence of a dialysable fraction in normal bovine whey capable of killing several species of bovine mycoplasmas. J. Hyg. 74:261-270.

4. Subcommittee on the Taxonomy of Mycoplasmatales. 1972. Proposal for minimal standards for descriptions of new species of the order Mycoplasmatales. Int. J. Syst. Bacteriol. 22:184-188. 